\title{
Déformabilité des sols non saturés à diverses températures
}

\author{
Unsaturated soil deformability \\ at varying temperatures
}

\author{
E. RECORDON \\ Professeur, Ecole Polytechnique Fédérale de Lausanne*
}

Rev. Franç. Géotech. n65, pp. 37-56 (octobre 1993)

\section{Résumé}

Cette publication donne les résultats de nombreux essais de déformabilité exécutés à l'appareil œdométrique sur deux types de sols non saturés: sable fin et limon. Elle montre comment évoluent les déformations sous charge constante au cours du temps, quelle est l'influence de la température dans le domaine $2^{\circ}$ à $40^{\circ}$ et quels sont les principaux paramètres qui influencent la déformabilité et la succion pour une température donnée.

\footnotetext{
Abstract

This publication presents the results from numerous deformability tests performed with oedometers on two different types of unsaturated soil : namely fine sand and silt. It describes how the deformations evolve with time under constant loading, what influence temperature has between $2^{\circ}$ and $40^{\circ}$ and what are the principal parameters which influence the deformability and suction for a given temperature.
} 


\section{INTRODUCTION}

La prévision du tassement des fondations superficielles peut être entachée d'une erreur importante si le niveau d'appui se trouve dans la zone non saturée, nettement au-dessus du niveau de la nappe souterraine (fig, 1).

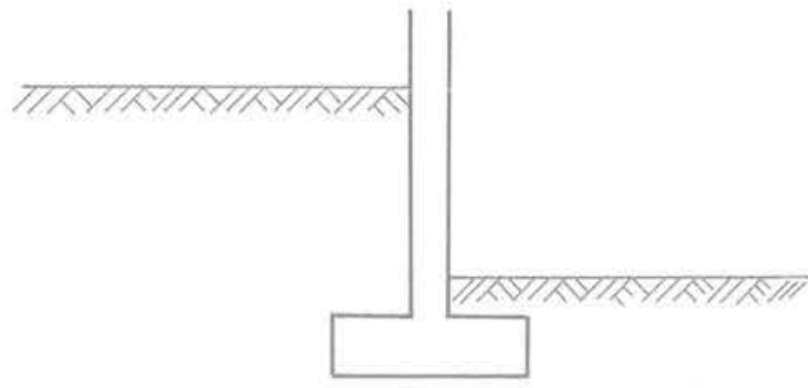

zone non saturée - succions

Non saturated zone - suction

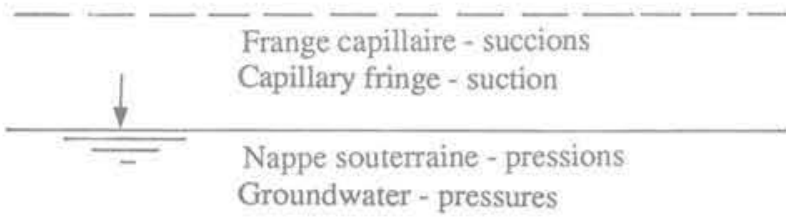

Figure 1.

L'essai œedométrique conçu pour étudier la déforma. bilité des sols saturés en consolidation primaire selon la théorie de TERZAGHI (1925) peut être utilisé, dans le cas des sols non saturés, comme le recommandent LAMBE et WITHMAN (1969), chap. 9. Dans ce cas, le calcul de la déformation est basé jusqu'ici sur la théorie de l'élasticité. Cela veut dire que seul le module de déformation est considéré. Aucune allusion n'est faite à l'évolution des déformations dans le temps. Dans le chapitre 9, consacré au « sol sec » (dry soil), les auteurs ne parlent pas des pressions interstitielles. Les raisonnements sont faits en contraintes totales, sans se préoccuper de connaître le rôle que pourrait jouer la succion sur le comportement du sol qui est manifestement non saturé, sans que cela soit dit explicitement.

Dans le domaine de l'agronomie et du génie rural, par contre, les recherches sur les sols non saturês ont fait l'objet d'ouvrages importants. Nous ne citerons que ceux de BAER $(1972,1979,1987)$ qui donnent les équations des écoulements biphasiques, eau-air, dans le but de modéliser les phénomènes de migration de l'eau, de l'air et des polluants, dans lesquels la succion, pression interstitielle négative, joue en général un rôle important. Dans ces études, une hypothèse simplificatrice est faite: la porosité est constante et ne dépend pas de la succion, le squelette minéral est indéformable, ce qui ne peut plus être admis si lon veut étudier les tassements des fondations.

Pour aboutir à une véritable mécanique des milieux non saturés, il faut examiner si les variations de porosité ont une influence sur la succion ou inversement. Il faut relier les travaux des mécaniciens du sol à ceux des spécialistes des écoulements polyphasiques. Cela reste à faire.

Cette publication est un premier pas dans cette direction. Il nous parait important tant du point de vue didactique que scientifique de dire que dans la zone non saturée, il est possible bien que difficile, d'envisager le comportement du sol de la même manière que l'a fait TERZAGHI dans le cas du sol saturé, mais en considérant les succions comme pressions interstitielles négatives.

Les nombreuses expériences qui ont été faites pour l'êtude de la pénétration du gel dans les sols ont mis en évidence l'influence très importante de la température sur les phénomènes de succion. C'est la raison pour laquelle nous avons d'emblée accordé une attention particulière à la température lors des expériences faites à l'œdomètre.

\section{LES SOLS ÉTUDIÉS}

Ces dernières années, plus de quatre-vingt essais œdométriques ont étê faits au Laboratoire de mécanique des sols de l'Ecole Polytechnique Fédérale de Lausanne sur des sols non saturés, à diverses températures et avec mesure de la succion par tensiomètre.

Les deux principaux sols étudiés sont:

- un sable fin,

- un limon.

La granulométrie et la classification de ces sols sont données à la figure 2 et au tableau 1 .

La granulométrie du sable est serrée $(U=3,7)$ alors que celle du limon est plus étendue $(U=20)$.

Les courbes de compactage à l'essai Proctor standard sont données sur la figure 3 . Elles montrent que le sable

Tableau 1.

Table 1

\begin{tabular}{|c|c|c|c|c|c|c|c|c|}
\hline \multirow{2}{*}{$\begin{array}{l}\text { Type } \\
\text { de sol }\end{array}$} & \multirow{2}{*}{$\begin{array}{l}\text { Classe } \\
\text { USCS }\end{array}$} & \multicolumn{4}{|c|}{ Fractions granulométriques (\%) } & \multicolumn{3}{|c|}{ Limites de consistance (\%) } \\
\hline & & Gravier & Sable & Limon & Argile & $W_{L}$ & $W_{p}$ & $\mathrm{I}_{\mathrm{p}}$ \\
\hline $\begin{array}{l}\text { Sable fin } \\
\text { Limon }\end{array}$ & $\begin{array}{l}\mathrm{SM} \\
\mathrm{CL}\end{array}$ & $\begin{array}{l}0 \\
8\end{array}$ & $\begin{array}{l}90 \\
13\end{array}$ & $\begin{array}{l}10 \\
62\end{array}$ & $\begin{array}{r}0 \\
17\end{array}$ & $\begin{array}{l}23,3 \\
31,4\end{array}$ & $\begin{array}{l}19,4 \\
19,6\end{array}$ & $\begin{array}{r}3,9 \\
11,8\end{array}$ \\
\hline
\end{tabular}




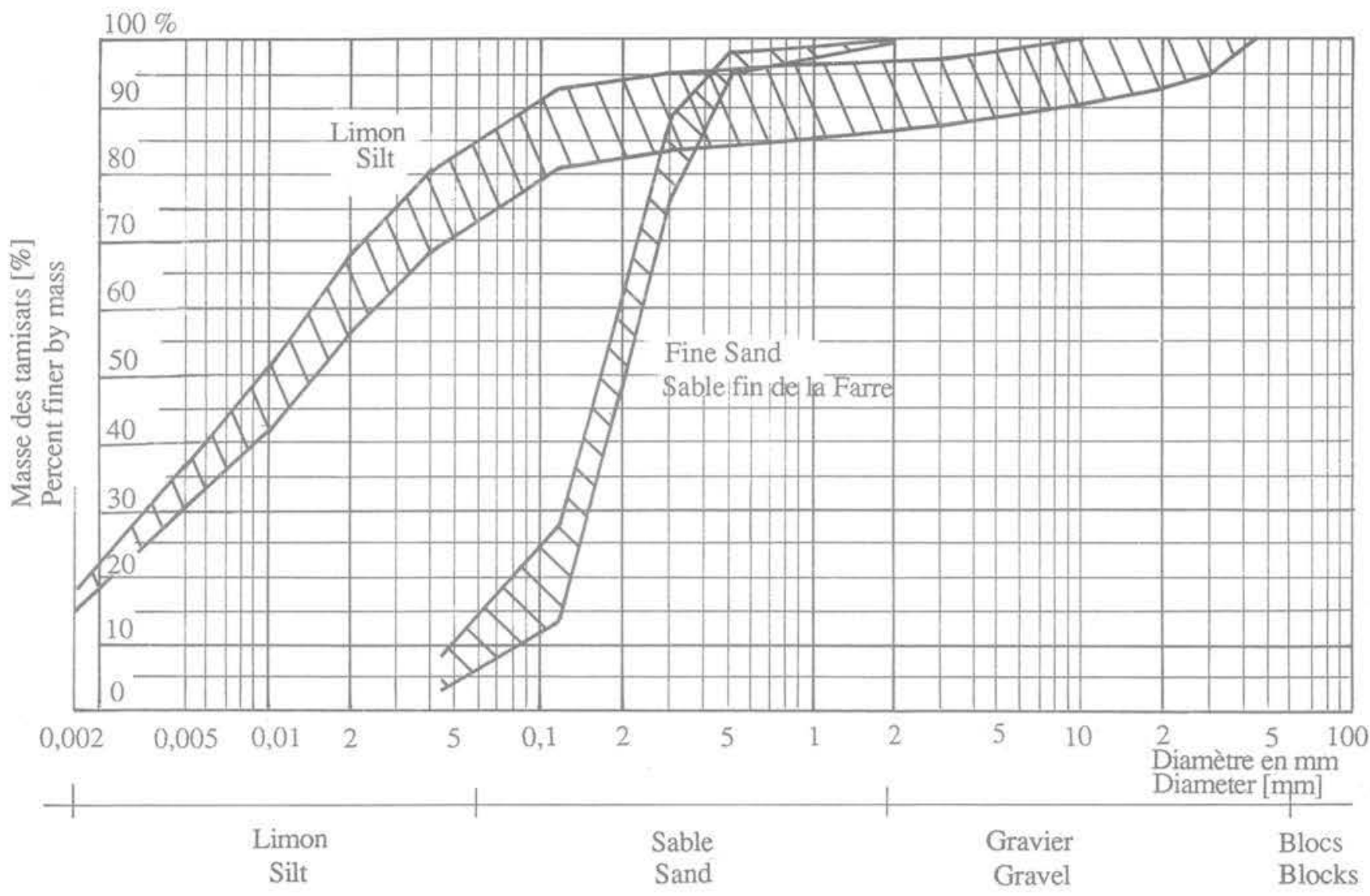

Fig. 2. - Granulométries.

Fig. 2. - Granulometry.

atteint une masse spécifique maximum $\rho_{j}=1,62 \mathrm{t} / \mathrm{m}^{3}$ pour une teneur en eau optimum de $15,7 \%$ environ, alors que le limon devient plus compact $\rho_{d}=1,87 \mathrm{t}$ / $\mathrm{m}^{3}$ pour $\mathrm{w}_{\text {opt }}=13,2 \%$.

\section{LES ESSAIS OEDOMÉTRIQUES}

La procédure de préparation des éprouvettes et d'exécution des essais oedométriques a été la suivante:

- mélange du sol sec et de l'eau en proportions déterminées par pesées de manière à obtenir la teneur en eau choisie;

- le sol ainsi préparé est placé dans l'œedomètre à son état le plus lâche, sans aucun compactage;

- mise en charge par paliers, comme dans les essais traditionnels. La charge appliquée correspond à une contrainte verticale de $4,12,60,125,250,500$ et $1000 \mathrm{kPa}$;

- lappareil œdométrique utilisé (fig. 4) est classique à l'exception des plaques généralement poreuses. La plaque supérieure ne l'est pas et permet de répartir la charge sur l'éprouvette. La base est munie d'un tensio. mètre pour la mesure des succions;

- celles-ci sont mesurées comme les déplacements verticaux (micromètres), pour chaque palier de charge, jusqu'à achèvement des déformations de l'éprouvette, ce qui se produit pratiquement après une durée d'un à trois jours selon le type de sol ;

- les appareils oedométriques sont placés dans une chambre climatisée à température réglable et constante pour un essai donné et à atmosphère saturée de vapeur d'eau. Les températures adoptées ont été $2^{\circ}, 20^{\circ}, 30^{\circ}$ et $40^{\circ}$.

Le jeu entre la paroi de l'cedomètre et la plaque de charge supérieure imperméable permet à l'air de s'évacuer. La teneur en eau, contrôlée au début et à la fin de l'essai ne varie pratiquement pas.

Le nombre d'essais exécutés est le suivant:

Tableau II.

Table II.

\begin{tabular}{|l|c|c|c|c|}
\hline \multirow{2}{*}{ Sol } & \multicolumn{4}{|c|}{ Température $\left({ }^{\circ} \mathrm{C}\right)$} \\
\cline { 2 - 5 } & $2^{\circ}$ & $20^{\circ}$ & $30^{\circ}$ & $40^{\circ}$ \\
\hline Sable fin 1 & 3 & 27 & 3 & 3 \\
Limon & 1 & 32 & - & 1 \\
Sable fin 2 & - & 13 & - & - \\
\hline
\end{tabular}

Parmi ces 83 essais, seuls 19 sur le sable fin 1 et 12 sur le limon ont été analysés pour cette publication car ils sont comparables, le programme de chargement 


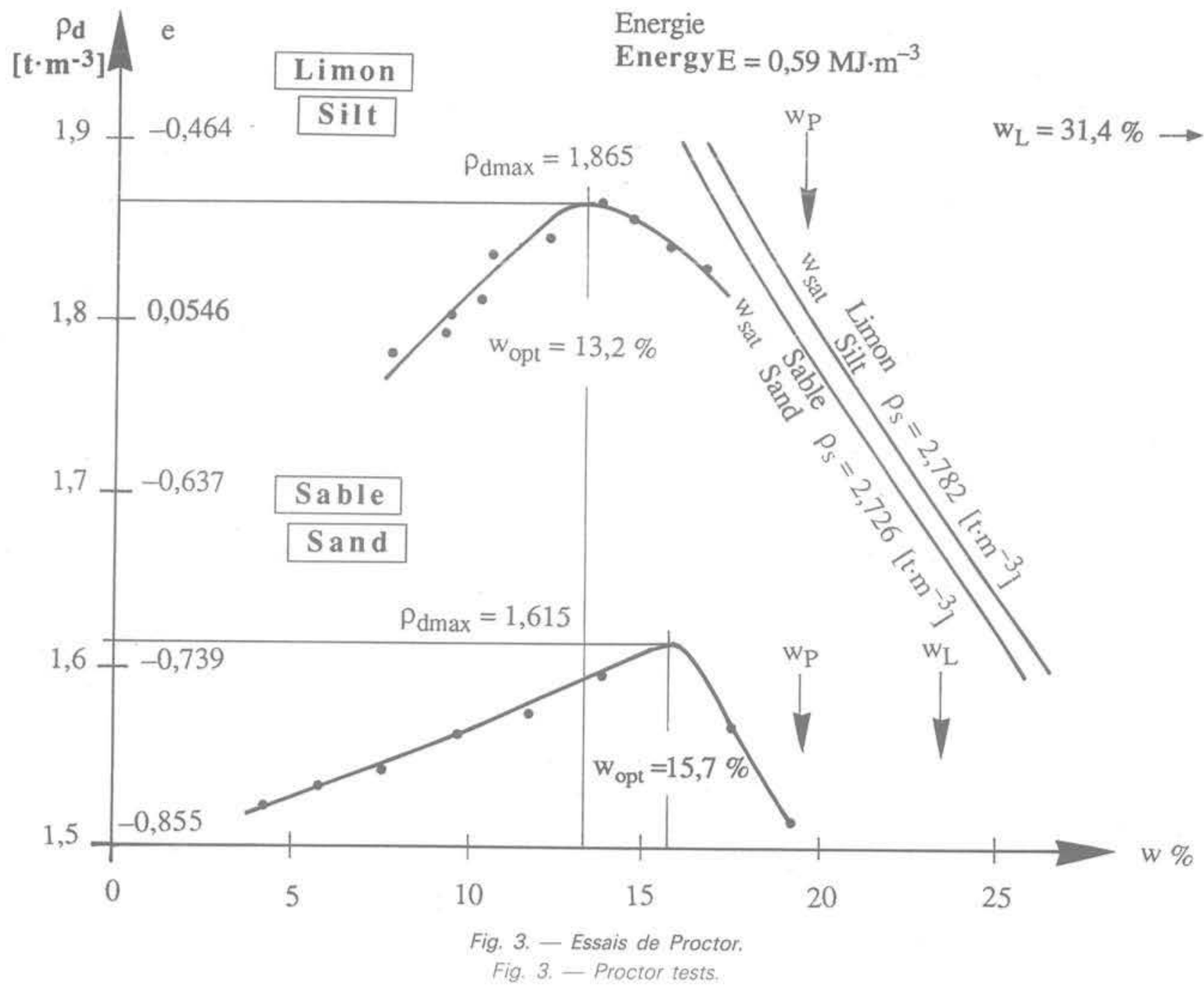

étant le même, Les autres essais ont été faits soit à des teneurs en eau très voisines pour contrôler la repro. ductibilité des résultats, qui est d'ailleurs très bonne, soit avec un autre programme de chargement, en particulier des cycles de charge - décharge dont il ne sera pas question ici.

\section{DÉFORMABILITÉ DES SOLS NON SATURÉS}

Les résultats donnés dans ce chapitre le sont sous forme de courbes œdométriques. L'indice de vide correspond aux déformations finales de chaque palier de charge. Toutefois, contrairement à l'habitude, les contraintes de compression $\sigma$ sont des contraintes totales et non effectives (échelle logarithmique).

L'indice de vide e, rapport entre la porosité du sol et le volume total de la matière minérale, est lié à la hauteur h de l'éprouvette par une relation linéaire

$$
h=\frac{M s}{\rho_{s} S}(1+e)=h_{s}(1+e)
$$

Ms [g] : masse de la matière minérale de l'éprouvette

$\rho_{\mathrm{s}}\left[\mathrm{g} \cdot \mathrm{cm}^{-3}\right]$ : masse spécifique de la matière minérale

$\mathrm{S}\left[\mathrm{cm}^{2}\right]$ : section horizontale de l'éprouvette

$h_{\mathrm{s}}[\mathrm{cm}]$ : hauteur qu'aurait l'éprouvette si elle était constituée de grains minéraux seulement (porosité nulle)

Les indices de vide pourraient donc être remplacés en ordonnée par la hauteur de l'éprouvette, en utilisant une échelle convenable.

Le module de déformation œedométrique augmente avec la contrainte ; il est donné par :

$$
E_{\text {oed }}=\frac{\Delta \sigma}{\Delta h / h}=\left(\sigma_{2}-\sigma_{1}\right) \frac{1+e_{1}}{e_{1}-e_{2}}[\mathrm{kPa}]
$$

On définit encore l'indice de compression, pente de la courbe œdométrique :

$$
C_{c}=\frac{e_{1}-e_{2}}{\log \left(\sigma_{2} / \sigma_{1}\right)}[-]
$$




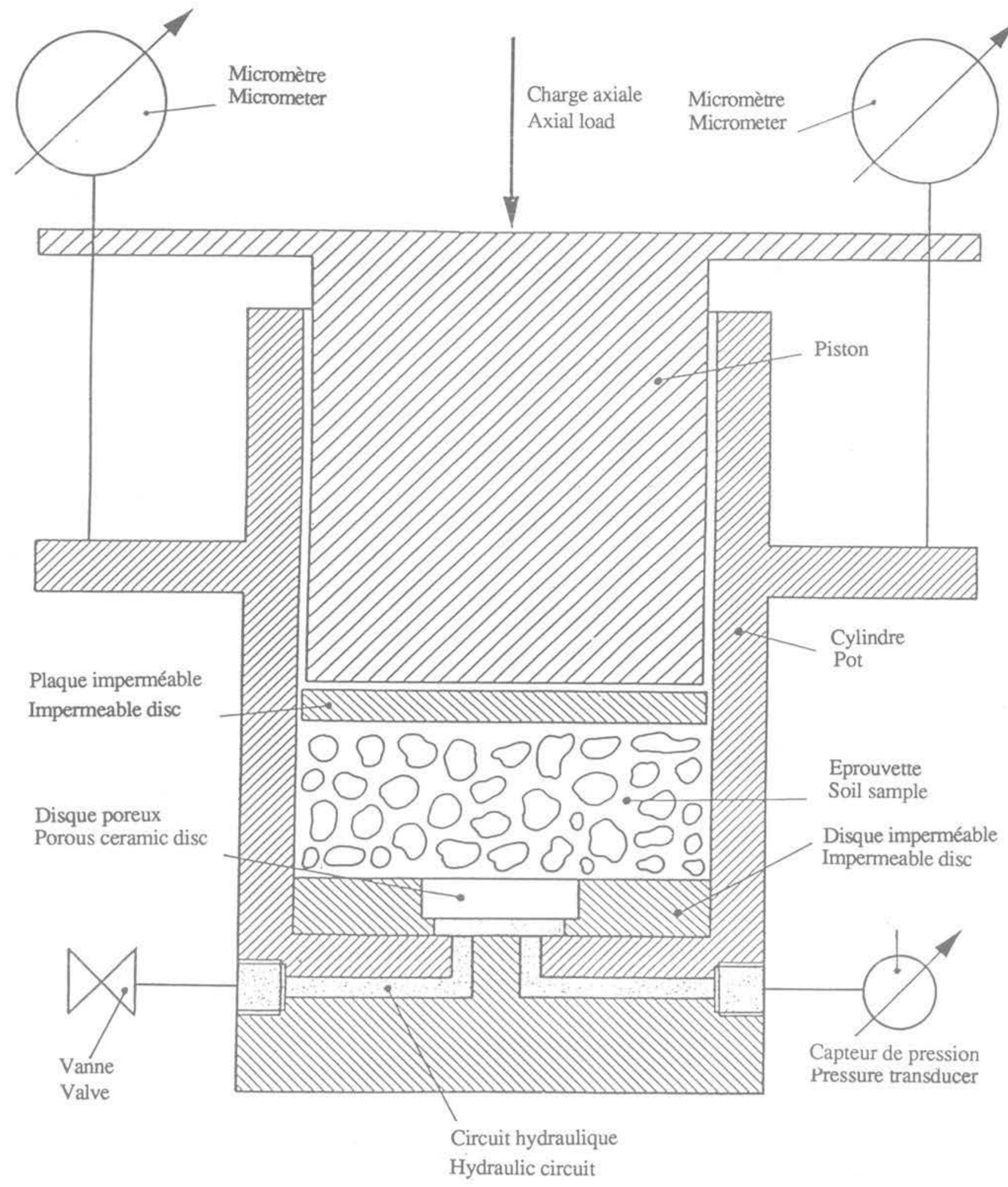

Fig. 4. - Edomètre.

Fig. 4. - Oedometer.

\subsection{Déformabilité du sable fin}

Les figures 5 à 8 représentent les courbes œdométriques. Les constatations suivantes peuvent être faites: - le sol étant non saturé pour les teneurs en eau de $2,5 \%$ à $20 \%,\left(S_{r}<65 \%\right)$, sa déformabilité est sensi- blement la même quelle que soit la teneur en eau. Les courbes œdométriques sont rectilignes dans l'intervalle $60<\sigma<1000 \mathrm{kPa}$ et ont même pente. Les plus faibles contraintes $(\sigma<60 \mathrm{kPa}$ ) correspondent à des surcharges de mise en place et d'homogénéisation de l'éprouvette par compactage. L'indice de compression 
Indice de vide

Void ratio

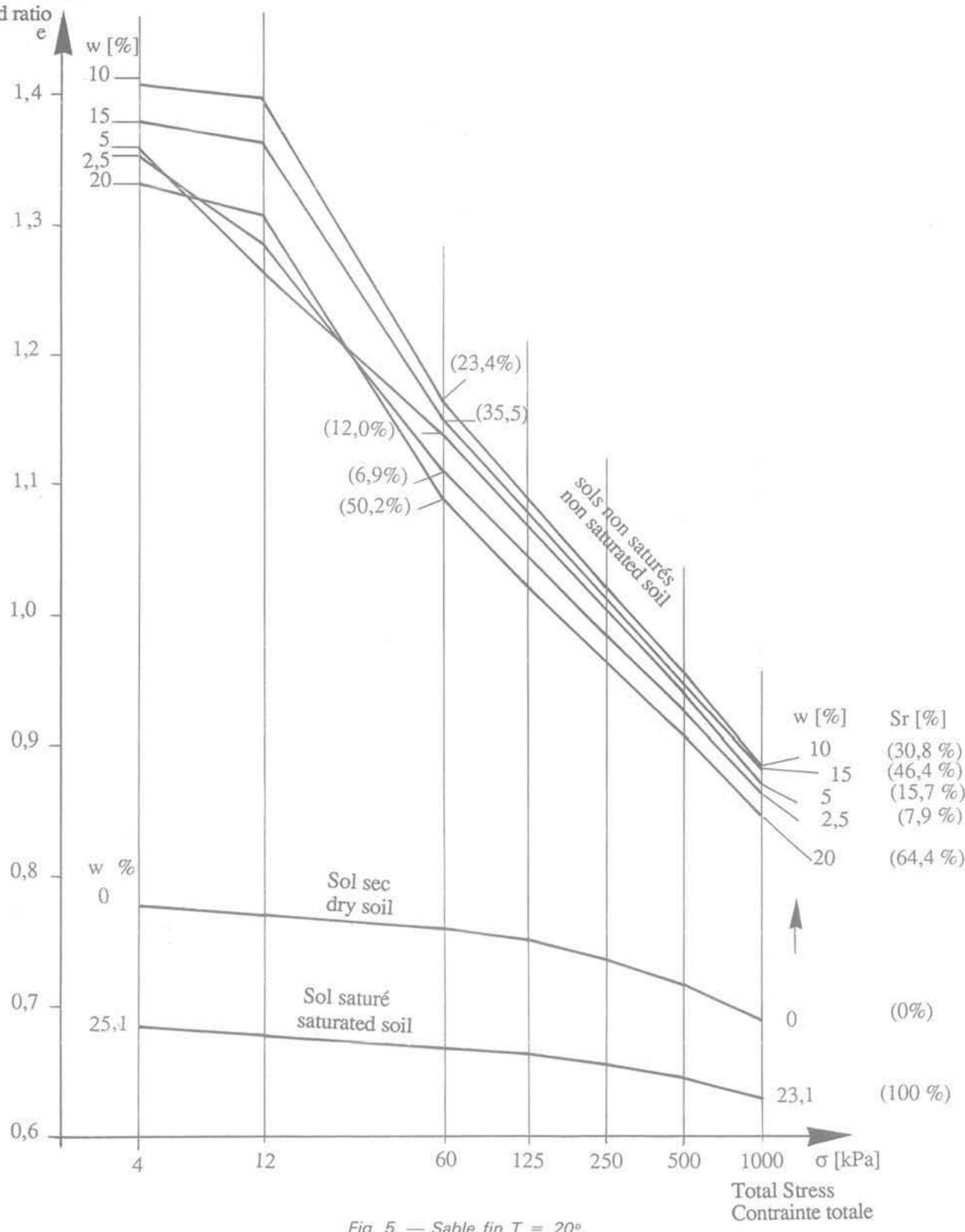

Fig. 5. - Sable fin $T=20^{\circ}$.

Contrainte totale

Fig. 5. - Fine sand $T=20^{\circ}$

et le module de déformation varient peu avec la teneur en eau. Le module de déformation dépend de la contrainte appliquée, comme le montre le tableau III dans le cas du sable fin non saturé;
- lorsque le sol est saturé ou sec, lors de sa mise en place, il atteint, sous son propre poids, une compacité beaucoup plus grande que s'il est partiellement saturé. La présence des deux phases eau et air, même si lune 


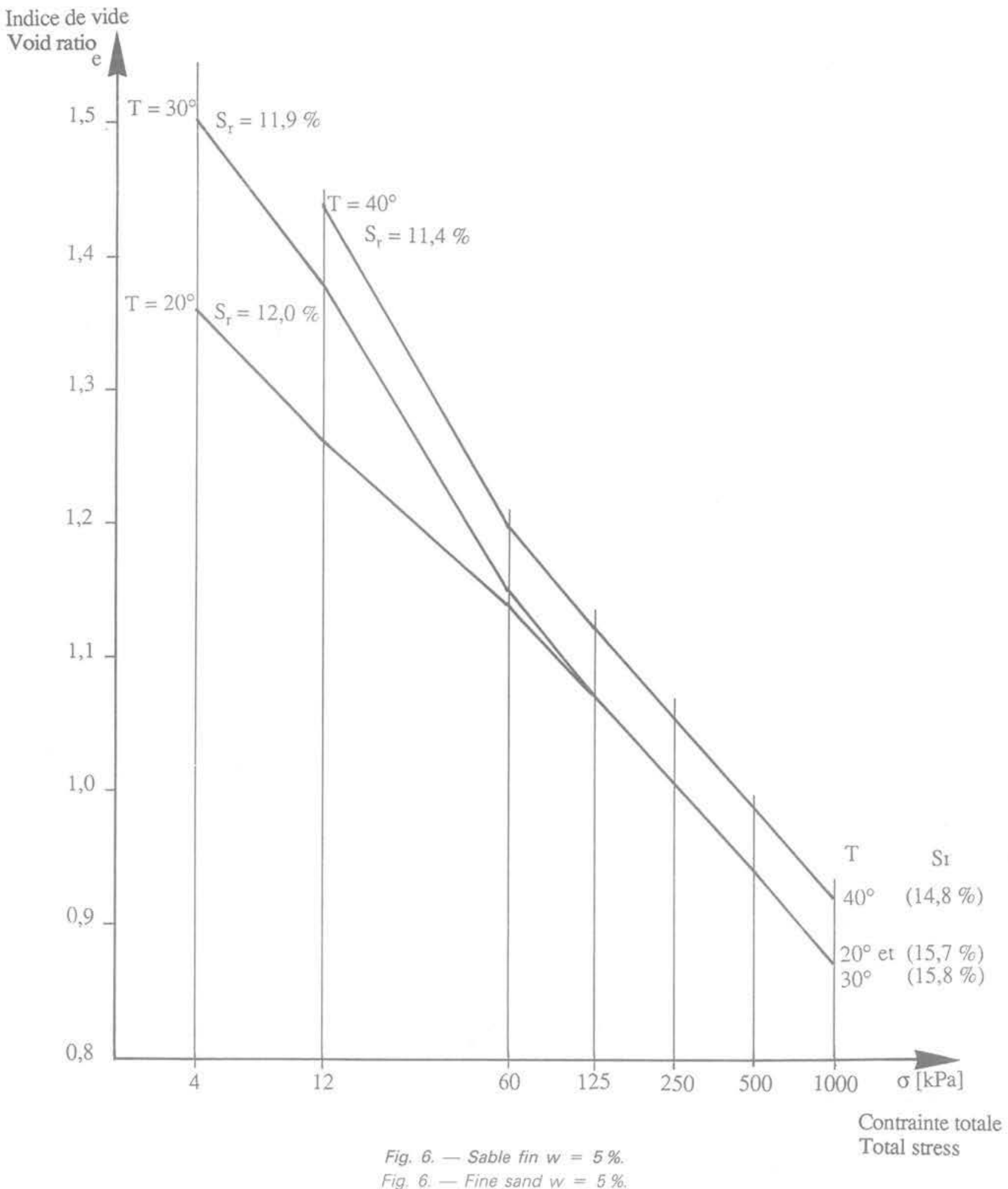

des deux n'est présente qu'en faible quantité, fait ap. paraître des forces capillaires qui empêchent le sol de se compacter avant surcharge. Le tableau IV donne les valeurs des caractéristiques de déformabilité pour le sable fin sec et saturé;

- pour une même teneur en eau, la température n'influence pratiquement pas la déformabilité du sable fin.
Le tableau $\mathrm{V}$ donne les valeurs extrêmes obtenues pour les onze essais exécutés avec $w=5 \%, 10 \%, 15 \%$ et pour $\mathrm{T}=2^{\circ}, 20^{\circ}, 30^{\circ}, 40^{\circ}$. Les écarts entre les valeurs extrêmes de $\mathrm{C}_{\mathrm{c}}$ est de $\mathrm{E}_{\text {oed }}$ restent faibles pour une même contrainte;

- la température sernble avoir un léger effet sur la compacité initiale. Dans tous les cas, l'indice de vide, 


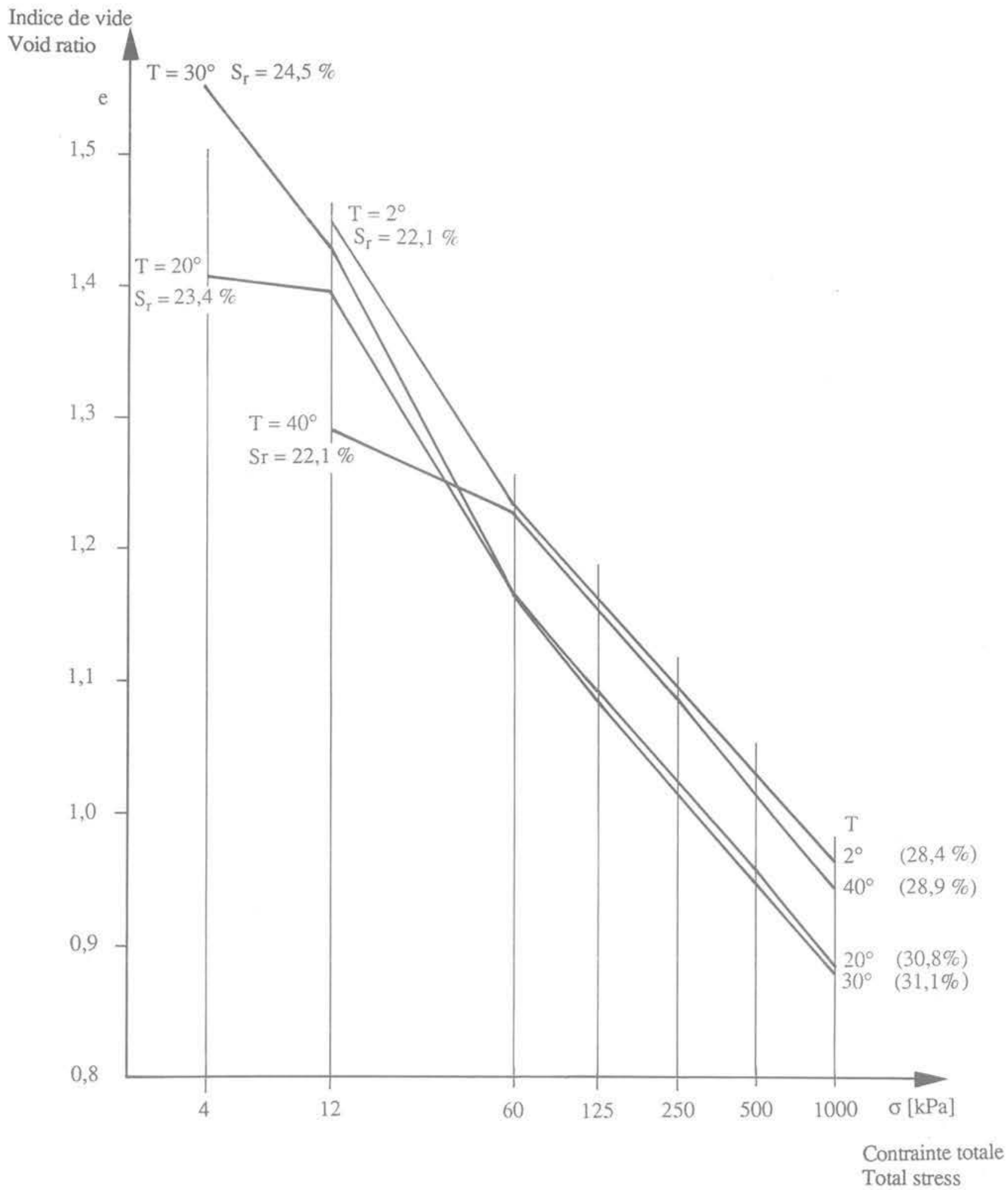

Fig. 7. - Sable fin $w=10 \%$.

Fig. 7. - Fine sand $w=10 \%$.

sous contrainte $\sigma=60 \mathrm{kPa}$, est plus faible à $20^{\circ}$ et $30^{\circ}$ que pour les températures de $2^{\circ}$ et $40^{\circ}$.

\subsection{Déformabilité du limon}

La préparation des éprouvettes de limon est beaucoup plus difficile que dans le cas du sable fin. Elle n'est plus possible lorsque la teneur en eau est inférieure à $10 \%$, le sol est hétérogène, les particules se liant en agglomérats.

La comparaison des courbes œdométriques du limon (fig. 9) et du sable fin (fig. 5 à 8) montre que, lors de la mise en place du sol dans l'appareil, le limon atteint d'emblée une compacité nettement plus grande que le sable. L'indice de vide du limon est compris entre 0,5 
Indice de vide

Void ratio

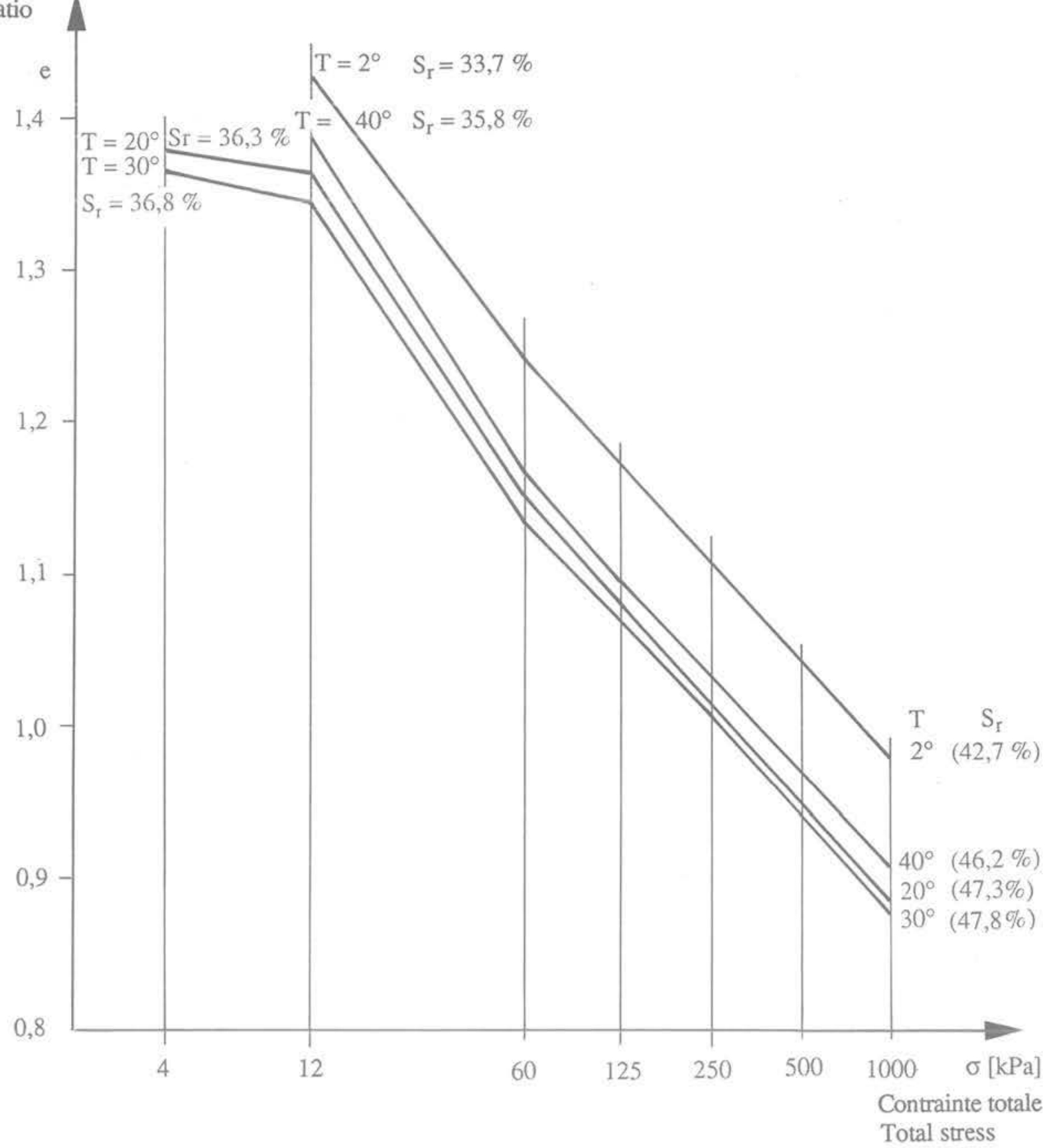

Fig. 8. - Sable fin $w=15 \%$.

Fig. 8. - Fine sand $w=15 \%$

Tableau III.

Table III.

\begin{tabular}{|c|c|c|c|}
\hline \multicolumn{2}{|c|}{$C_{\mathrm{c}}(-)$} & \multicolumn{2}{c|}{$\mathrm{E}_{\text {oed }}(\mathrm{kPa})$} \\
\hline $60<\sigma<125$ & $500<\sigma<1000$ & $60<\sigma<125$ & $500<\sigma<1000$ \\
\hline $\min : 0,204$ & $\min : 0,209$ & $\max : 2118$ & $\max : 15175$ \\
$\max : 0,245$ & $\max : 0,249$ & $\min : 1815$ & $\min : 12995$ \\
\hline
\end{tabular}

et 0,8 alors que celui du sable est de l'ordre de 1,3 à 1,5. C'est dire que la masse volumique apparente sèche est en moyenne $p_{\mathrm{d}}=1,69 \mathrm{t} / \mathrm{m}^{3}$ pour le limon et $p_{\mathrm{d}}=$ $1,14 \mathrm{t} / \mathrm{m}^{3}$ pour le sable. Cela s'explique par la différence de granulométrie. Le même phénomène se produit à l'essai de Proctor (fig. 3).

Le tableau VI donne les valeurs minimums et maximums de $\mathrm{C}_{\mathrm{c}}$ et de $\mathrm{E}_{\text {oed }}$ pour le limon. 
Tableau IV.

Table IV

\begin{tabular}{|l|c|c|c|c|}
\hline \multirow{2}{*}{} & \multicolumn{2}{|c|}{$\mathrm{C}_{\mathrm{c}}(-)$} & \multicolumn{2}{c|}{$\mathrm{E}_{\text {ood }}(\mathrm{kPa})$} \\
\cline { 2 - 5 } & $60<\sigma<125$ & $500<\sigma<1000$ & $60<\sigma<125$ & $500<\sigma<1000$ \\
\hline Sol sec $\mathrm{w}=0$ & 0,0345 & 0,0897 & 10114 & 32148 \\
Sol saturé & 0,0125 & 0,0532 & 25311 & 50876 \\
\hline
\end{tabular}

Indice de vide

Void ratio
Degré de saturation $\mathrm{Sr}$

Degree of saturation $\mathrm{Sr}$

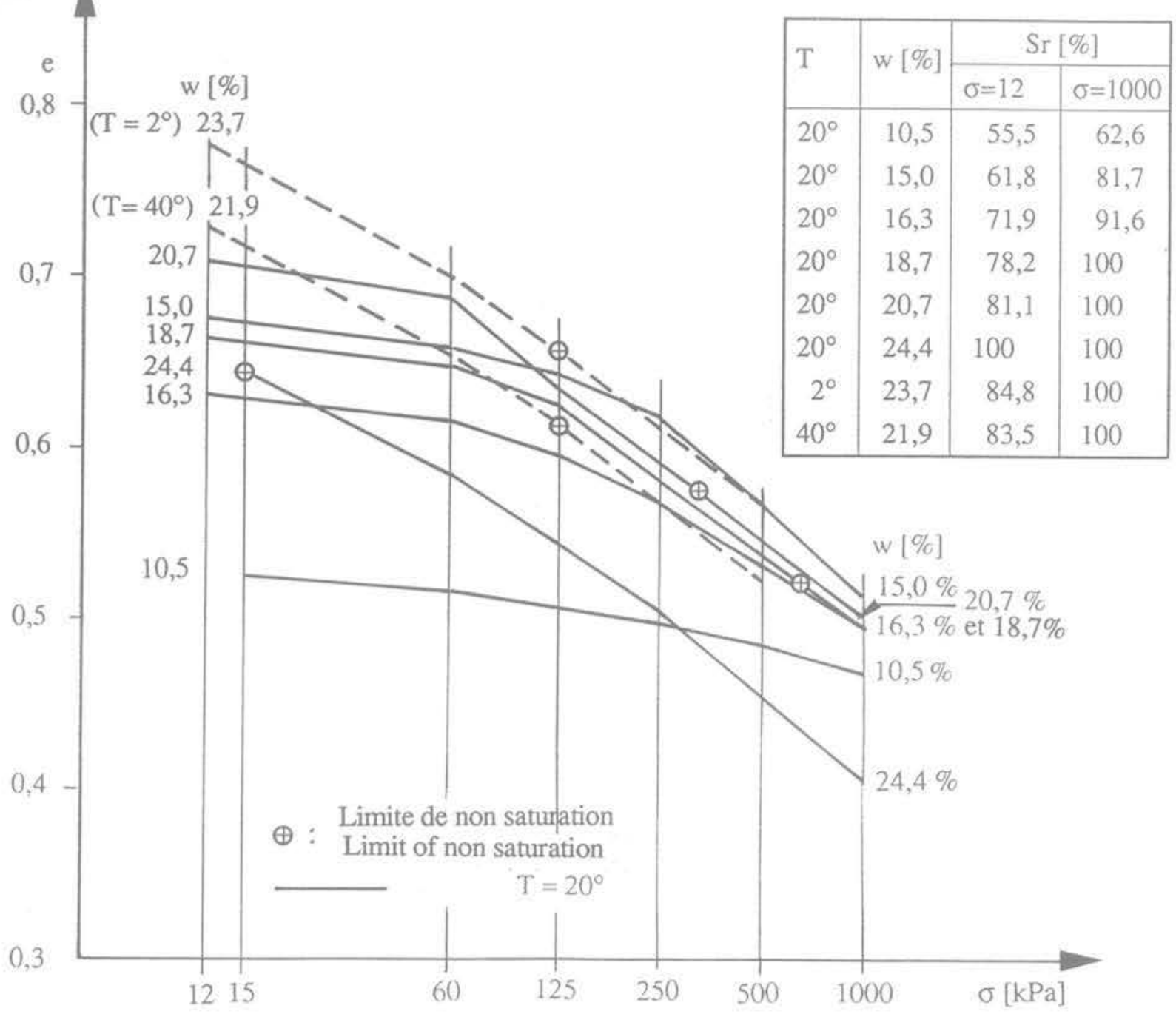

Fig. 9. - Limon.

Contrainte totale Total stress

Fig. 9. - Silt Total stress.

Ces valeurs peuvent être comparées à celles du tableau IV (sable).

Les valeurs de la première ligne correspondent à la teneur en eau la plus faible, $w=10,5 \%$, et la deuxième ligne à une teneur en eau élevée, $w=$
$20,7 \%$. Le limon est donc de plus en plus déformable lorsque la teneur en eau et le degré de saturation crois. sent.

Les deux essais exécutés à des températures de $2^{\circ}$ et $40^{\circ}$ (lignes tiretées de la fig. 9) montrent que l'effet de 
Tableau V.

Table V.

\begin{tabular}{|c|c|c|c|}
\hline \multicolumn{2}{|c|}{$C_{c}(-)$} & \multicolumn{2}{c|}{$E_{\text {ood }}(\mathrm{kPa})$} \\
\hline $60<\sigma<125$ & $500<\sigma<1000$ & $60<\sigma<125$ & $500<\sigma<1000$ \\
\hline $\min : 0,210$ & $\min : 0,176$ & $\max : 2164$ & $\max : 15087$ \\
$\max : 0,248$ & $\max : 0,239$ & $\min : 1781$ & $\min : 13224$ \\
\hline
\end{tabular}

Tableau VI.

Table VI.

\begin{tabular}{|c|c|c|c|}
\hline \multicolumn{2}{|c|}{$C_{c}(\cdot)$} & \multicolumn{2}{c|}{$E_{\text {oed }}(\mathrm{kPa})$} \\
\hline $60<\sigma<125$ & $500<\sigma<1000$ & $60<\sigma<125$ & $500<\sigma<1000$ \\
\hline $\min : 0,035$ & $\min : 0,060$ & $\max : 9158$ & $\max : 39576$ \\
$\max : 0,176$ & $\max : 0,169$ & $\min : 1978$ & $\min : 16976$ \\
\hline
\end{tabular}

la température est ici également négligeable pour des teneurs en eau élevées.

Notons finalement que les points marqués d'un cercle sur la figure 9 correspondent à la contrainte et à l'indice de vide pour lesquels le sol atteint la saturation par compression. La quantité d'eau restant pratiquement constante au cours de l'essai, si la teneur en eau est grande, le sol se sature par diminution du volume des interstices. L'air est alors entièrement évacué.

Seuls les trois essais à faibles teneurs en eau : 10,5, 15,0 et $16,3 \%$ correspondent à un état non saturé jusqu'à la fin du chargement.

\section{SUCCIONS}

Les résultats des mesures de succion, faites au cours des essais œedométriques sont représentés sur les figures 10,11 et 12 .

Ces figures montrent que la succion est influencée par les variations de porosité d'une part et par la température d'autre part.

Les figures 10 et 11 concernent les essais exécutés à $20^{\circ}$ sur le sable fin et sur le limon respectivement. Dans le cas du sable, la succion augmente avec la contrainte, donc lorsque le volume des interstices diminue. Cela est conforme à la loi de JURIN qui montre que les forces capillaires sont d'autant plus grandes que les tubes capillaires sont petits.

Par contre, la succion diminue lorsque le degré de saturation, c'est-à-dire la teneur en eau, augmente. Remarquons que pour tous les essais exécutés avec le sable, le degré de saturation est resté inférieur à $65 \%$ même sous les plus grandes charges.

Dans le cas du limon (fig. 11), les succions sont plus grandes que pour le sable, le sol étant plus fin.
Pour les raisons évoquées au chapitre précédent, il n'a pas été possible de faire des essais à des teneurs en eau inférieures à $10 \%$. Les degrés de saturation sont donc toujours supérieurs à $60 \%$. D'une manière générale, les succions décroissent avec la contrainte de compression, contrairement à ce que nous avons vu pour le sable faiblement saturé.

La diminution de la dimension des capillaires est donc contrecarrée par l'augmentation du degré de saturation car les succions doivent s'annuler lorsque le sol est saturé, ce que montre la figure 11.

On peut dire que lorsqu'il y a diminution de la taille des interstices, à teneur en eau constante, dans une première phase la succion augmente, elle atteint un maximum pour un degré de saturation de l'ordre de 60 à $70 \%$ puis elle diminue pour s'annuler lorsque $\mathrm{Sr}=100 \%$.

La figure 12 montre que la succion augmente avec la température, ceci naturellement d'autant plus que sa valeur est plus élevée, donc que la teneur en eau est plus faible. Cette figure ne concerne que le sable fin, les essais à d'autres températures étant trop peu nom. breux pour le limon.

\section{6. ÉVOLUTION DES DÉFORMATIONS DANS LE TEMPS}

Pour chacun des nombreux essais exécutés, une courbe d'évolution des tassements au cours du temps a été déterminée pour chacune des charges appliquées. Nous n'avons analysé que les résultats correspondant aux contraintes $\sigma=60,125,250,500$ et $1000 \mathrm{kPa}$. Cela représente néanmoins environ 150 courbes $\Delta h=f(t)$.

La loi de fluage généralement admise dans un tel cas, en logarithme du temps, correspond à une vitesse de déformation décroissante en $1 / \mathrm{t}$. Nous l'avons choisie et avons constaté qu'elle traduit très fidèlement les résultats expérimentaux:

$$
\begin{aligned}
\varepsilon_{1} & =\varepsilon_{20}+a \log t / t_{20} \quad \text { avec } \varepsilon_{1}=\frac{h_{0}-h_{1}}{h_{\circ}}=\frac{\Delta h_{1}}{h_{\circ}} \\
\text { ou } \Delta h_{1} & =\Delta h_{20}+h_{0} a \log t / t_{20}
\end{aligned}
$$

Dans ces équations les indices $i$ se réferent au temps exprimé en minutes: par exemple: $\varepsilon_{20}$ est la déformation verticale après 20 minutes, nous l'appellerons la déformation instantanée. $\Delta h_{20}$ est le tassement instantané, après 20 minutes. $h_{0}$ est la hauteur initiale de l'éprouvette au temps $t_{i}=0$ ou celle de la couche sollicitée en déformation dans le terrain.

$a$ est le coefficient de fluage. Il est sans dimensions. Avec $\varepsilon_{20}$ il caractérise le comportement du sol.

Le tableau VII donne les valeurs de $\varepsilon_{20}$ et de a dans les quatre cas extrêmes que nous avons trouvés parmi les 150 examinés. Les courbes de tassement correspondantes sont reproduites sur la figure 13 . 


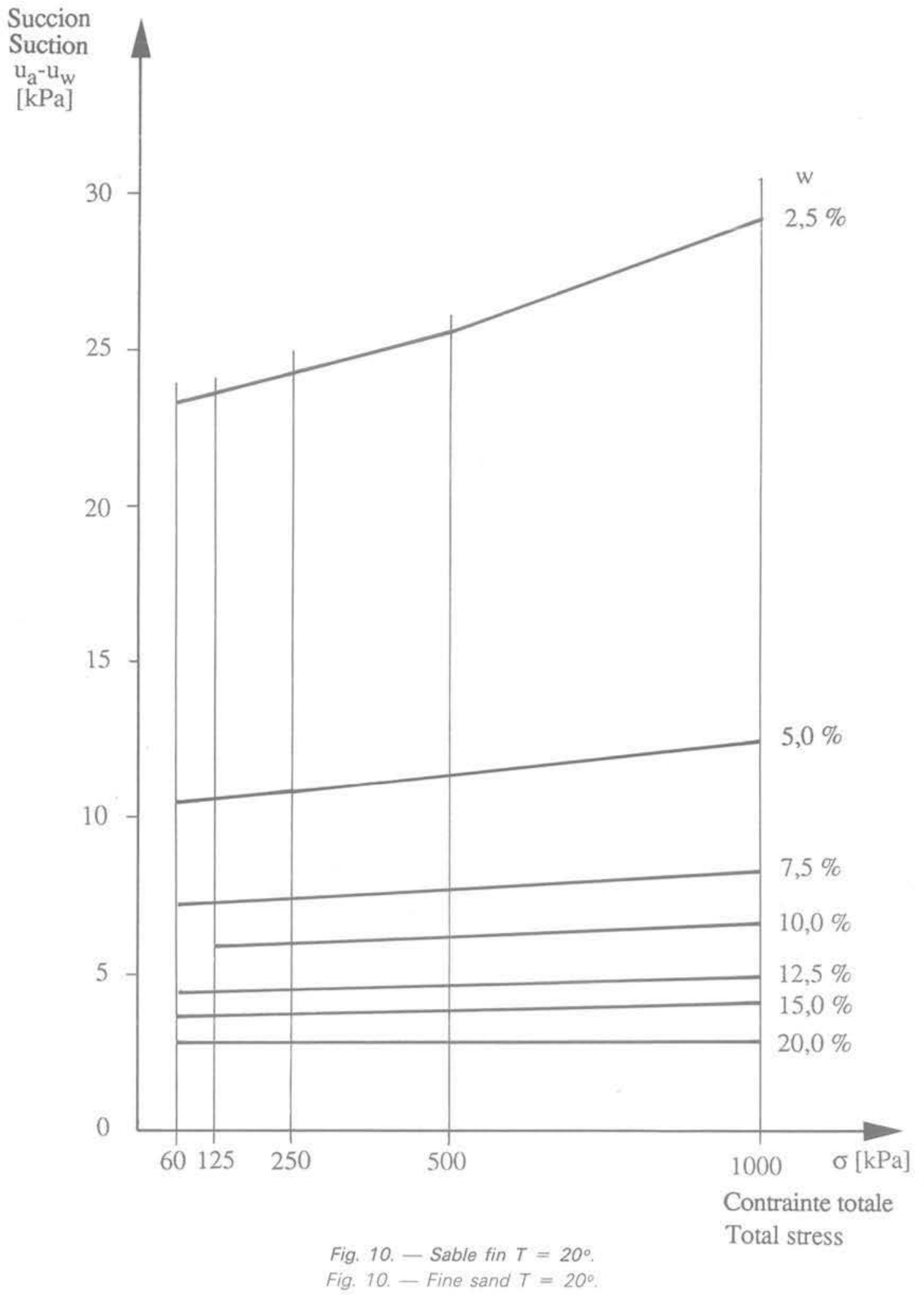

La loi de fluage en logarithme du temps a les défauts mathématiques suivants:

- pour $\varepsilon_{1}=0, t \neq 0$, alors qu'il devrait être nul. Dans ce cas, la relation $\log t_{1}=\log t_{20}-\frac{\varepsilon_{20}}{a}$ (avec log $t_{20}=\log 20$ [minutes] $=1,301$ ) permet de calculer les valeurs de $t$ données dans la dernière colonne du tableau VII pour $\varepsilon=0$. Ce défaut de la loi théorique peut être accepté car $t$ est toujours très petit pour $\varepsilon_{1}=0 . \mathrm{t}$ ne s'annule que pour $\varepsilon_{1}=-\infty$;

- pour $\mathrm{t}=\infty, \varepsilon=\infty$, contrairement à la loi de consolidation de TERZAGHI selon laquelle le tassement pour une durée infinie prend une valeur finie (assymp. tote horizontale). Toutefois, cette particularité théorique 


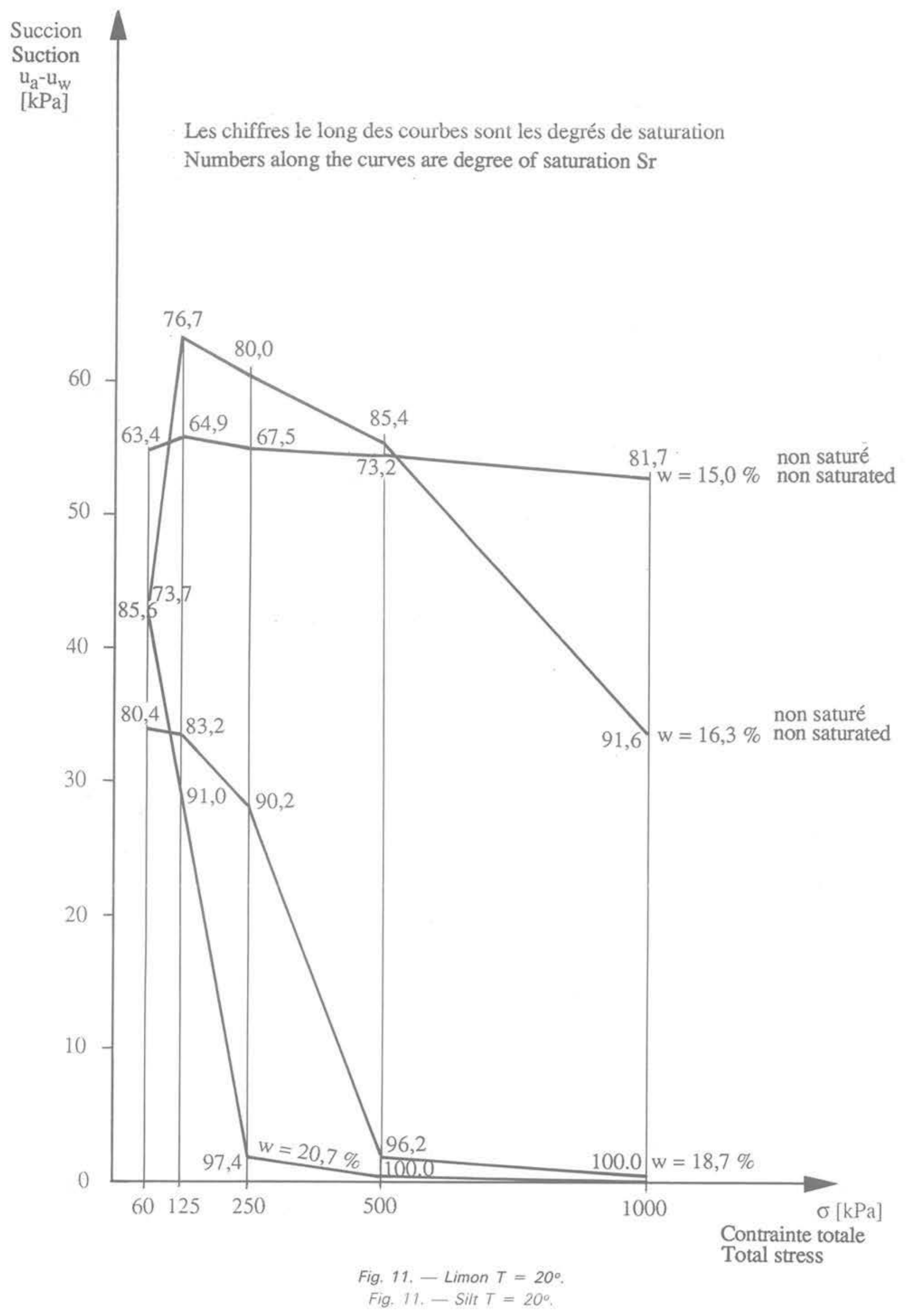


Tableau VII.

Table VII

\begin{tabular}{|c|c|c|c|c|c|c|c|c|}
\hline Cas & Sol & $\begin{array}{c}T \\
\left(^{\circ}\right)\end{array}$ & $\begin{array}{c}w \\
(\%)\end{array}$ & $\begin{array}{c}\sigma \\
(\mathrm{kPa})\end{array}$ & $\varepsilon_{20}$ & a & $\frac{\varepsilon_{20}}{a}$ & $\begin{array}{l}\mathrm{t}(\min ) \\
\mathrm{pr} \mathrm{s}_{1}=0\end{array}$ \\
\hline $\begin{array}{ll}1 & \varepsilon_{20 \max } \\
2 & \varepsilon_{20 \min } \\
3 & a_{\max } \\
4 & a_{\min }\end{array}$ & $\begin{array}{l}\text { Sable fin } \\
\text { Limon } \\
\text { Sable fin } \\
\text { Sable fin }\end{array}$ & $\begin{array}{r}30^{\circ} \\
2^{\circ} \\
40^{\circ} \\
2^{\circ}\end{array}$ & $\begin{array}{r}15,0 \\
23,7 \\
10,0 \\
5,0\end{array}$ & $\begin{array}{l}1000 \\
1000 \\
1000 \\
125\end{array}$ & $\begin{array}{l}0,035 \\
0,003 \\
0,012 \\
0,012\end{array}$ & $\begin{array}{l}0,0028 \\
0,0028 \\
0,0030 \\
0,0005\end{array}$ & $\begin{array}{r}12,5 \\
1,1 \\
4,0 \\
24,0\end{array}$ & $\begin{array}{l}6.10^{-12} \\
1.7 \\
0.002 \\
2.10^{-23}\end{array}$ \\
\hline
\end{tabular}

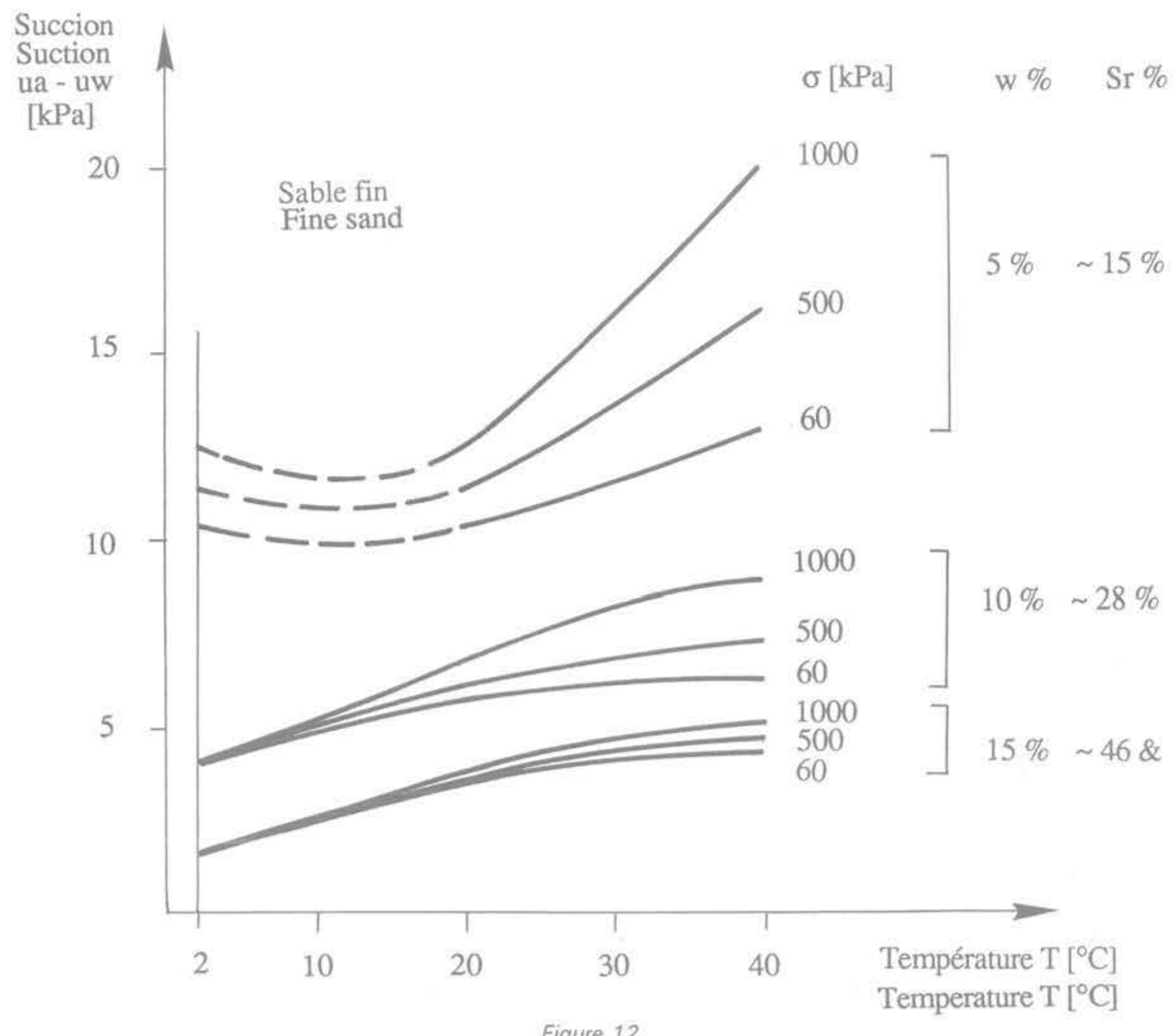

n'a pas de conséquence pratique, car la durée d'exis. tence d'un ouvrage de génie civile n'excède guère 100 ans.

Le tableau VIII donne les valeurs de $\varepsilon_{20}$, déformation atteinte durant les 20 premières minutes et de a log $\frac{t_{100 \text { ans }}}{t_{20}}$, le tassement correspondant à la période $t_{20}$

comprise entre $20 \mathrm{mn}$ et 100 ans, pour les exemples du tableau VII :
Tableau VIII.

Table VIII.

\begin{tabular}{|c|c|c|c|}
\hline Cas & $\varepsilon_{20}=\frac{\Delta h_{20}}{h_{0}}$ & $a \log \frac{t_{100 \text { nns }}}{t_{20}}$ & $\varepsilon_{i}$ \\
\hline 1 & 0,035 & 0,018 & 0,053 \\
2 & 0,003 & 0,018 & 0,021 \\
3 & 0,012 & 0,019 & 0,031 \\
4 & 0,012 & 0,003 & 0,015 \\
\hline
\end{tabular}


Tassements durant le premier jour

Settlements during the first day

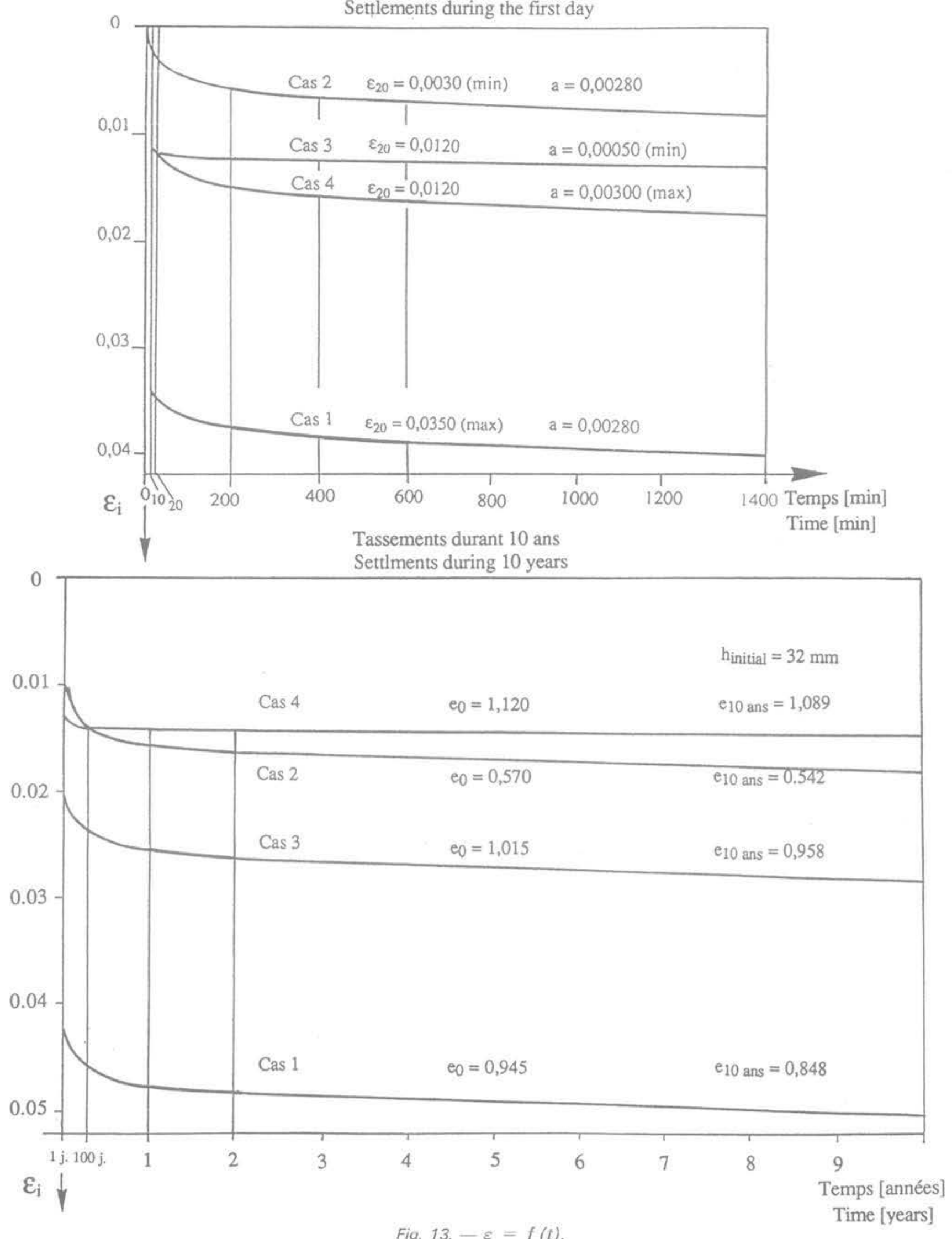



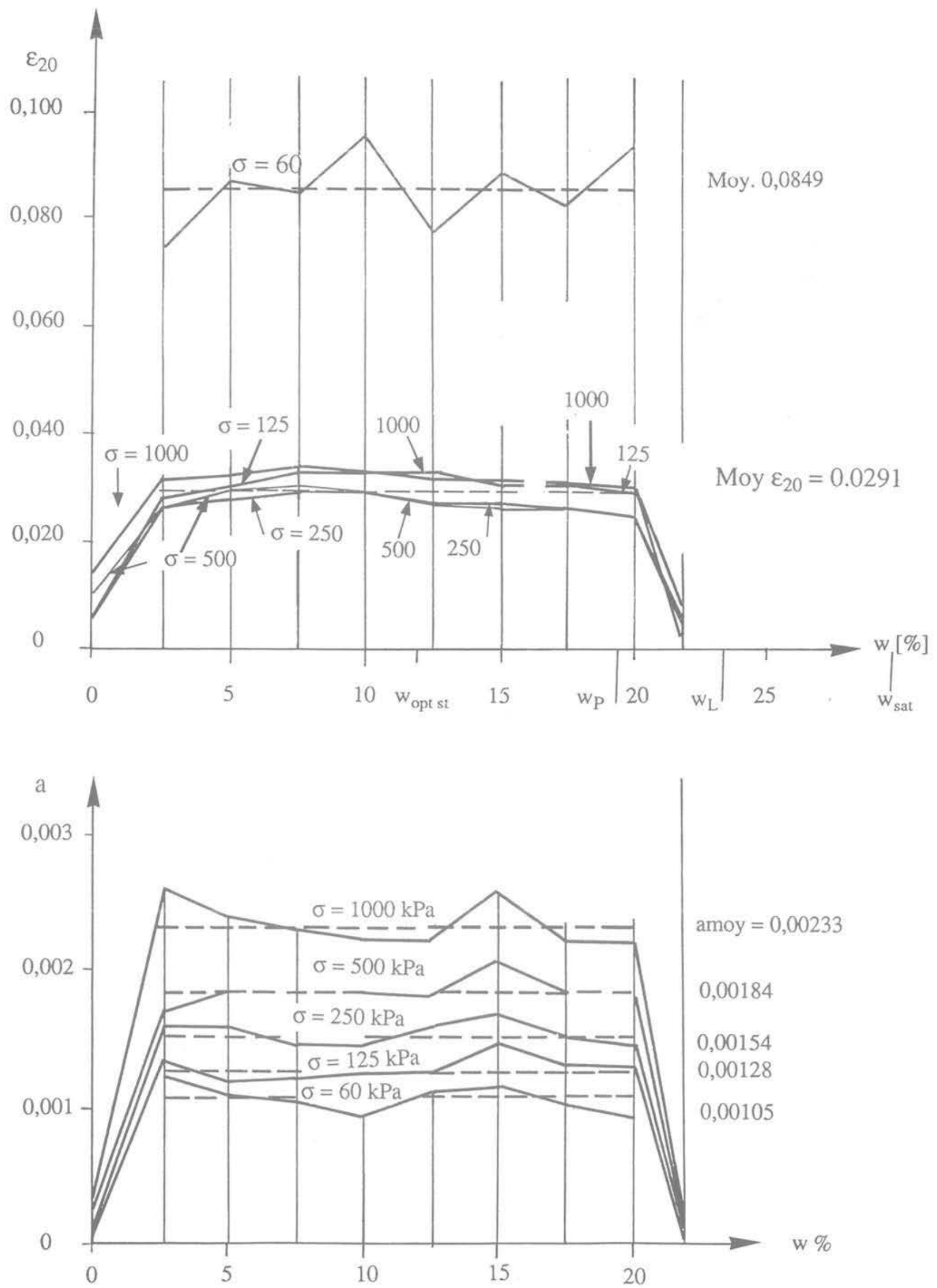

Fig. 14. - Sable fin $T-20$

Fig. 14. - Fine sand $T=20$. 
Dans le cas 1 , celui pour lequel les tassements sont les plus grands, le tassement instantané $\Delta \mathrm{h}_{20}=3,5 \%$, soit $35 \mathrm{~cm}$ pour $\mathrm{h}=10 \mathrm{~m}$. Le tassement entre 20 minutes et 100 ans est égal à $1,8 \%$ de $h_{\circ}$ ou $18 \mathrm{~cm}$. Pour la
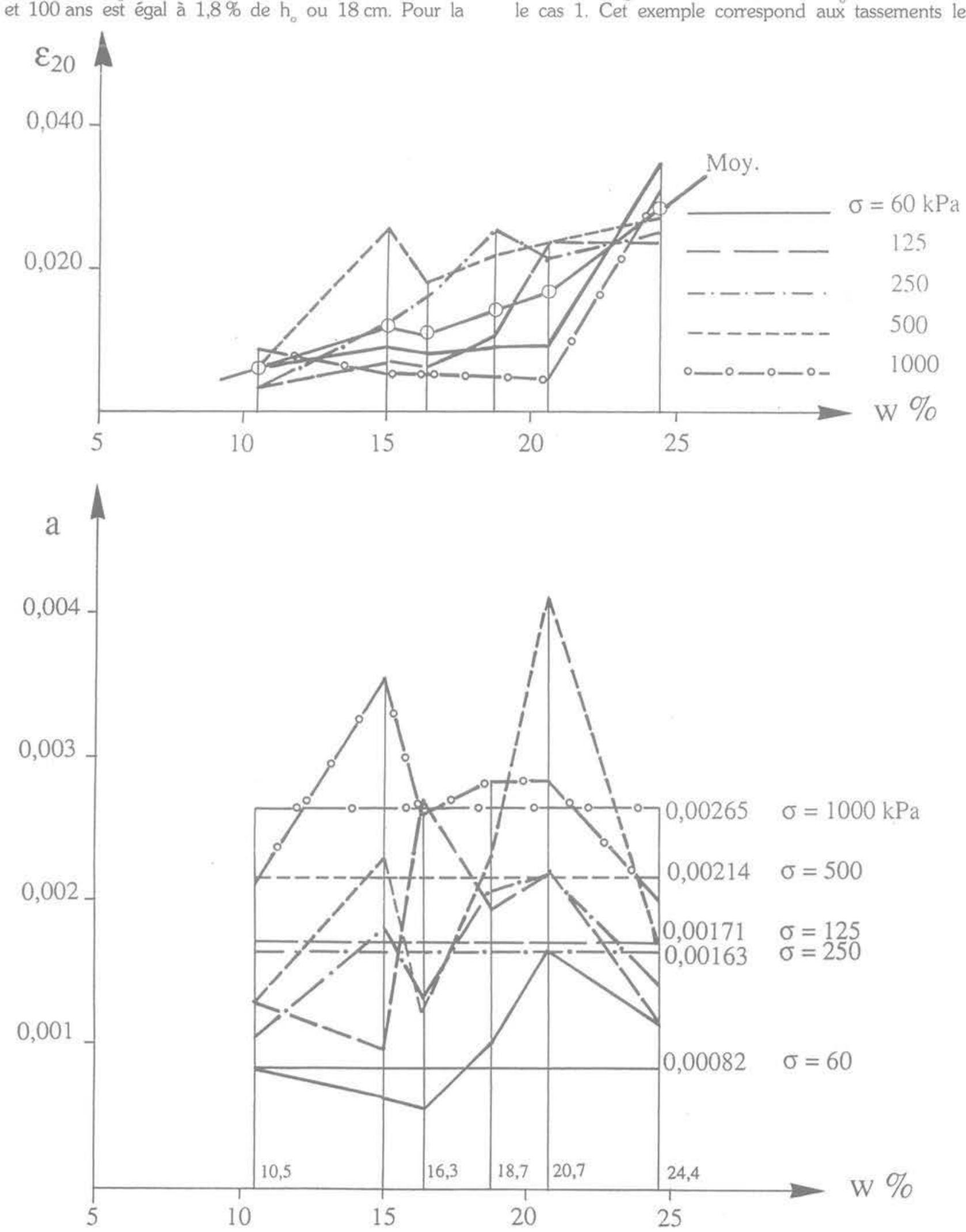

Fig. 15. - Limon $T=20^{\circ}$

Fig. $15 .-$ Silt $T=20^{\circ}$. période de 100 ans à 1000 ans, le rapport $t / t_{20}=10$, son logarithme est égal à 1 , et l'augmentation de tassement est égale à a, soit $0,3 \%$ de $\mathrm{h}$ ou $3 \mathrm{~cm}$ dans le cas 1. Cet exemple correspond aux tassements les 

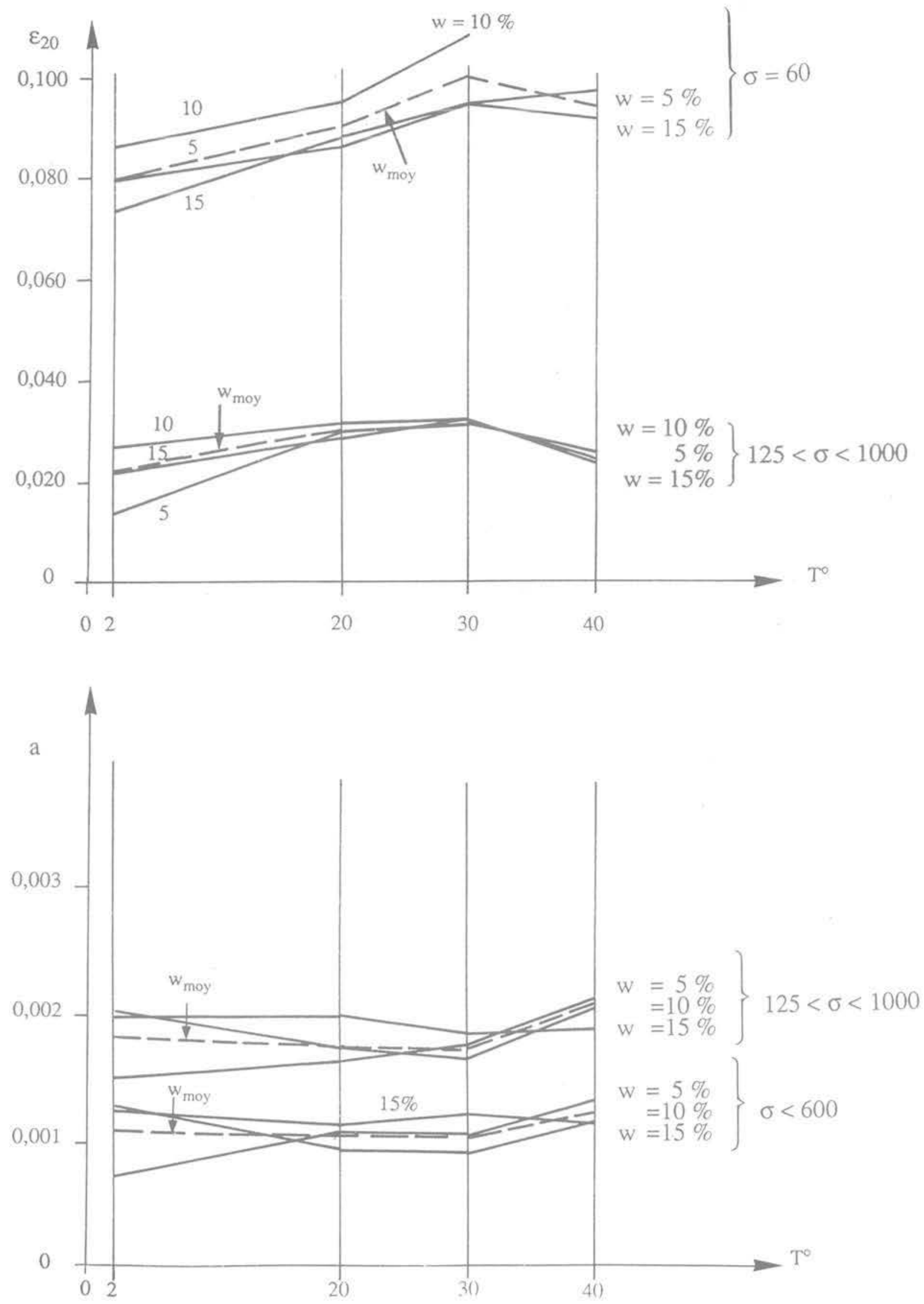

Fig. 16. - Sable fin.

Fig. 16. - Fine sand. 
plus grands que l'on peut avoir dans le cas du sable. Ils restent faibles. Le tassement total est de l'ordre de $5 \%$ de l'épaisseur de la couche comprimée.

Les figures 14 et 15 montrent comment varient $\varepsilon_{20}$ et a en fonction de $w$ et de la contrainte verticale $\sigma$. Dans le cas du sable, fig. 14, si l'on exclut les cas extrêmes du sol sec et du sol saturé, les deux paramètres ne varient pas quelle que soit la teneur en eau. L'influence de $\sigma$ sur $\varepsilon_{20}$ est aussi nulle, si l'on excepte la valeur $\sigma=60 \mathrm{kPa}$. Le paramètre a augmenté régulièrement avec $\sigma$.

Dans le cas du limon, fig. 15, les valeurs sont plus dispersées. On note toutefois la tendance à une croissance des valeurs moyennes de $\varepsilon_{20}$ avec $w$. Cette variation est sensiblement la même quel que soit $\sigma$. Le paramètre présente une variation du même type que pour le sable : constance en fonction de w et augmentation avec $\sigma$.

La figure 16, relative au sable fin montre que les deux paramètres sont peu influencés par la température. Seul $\varepsilon_{20}$, dans le domaine de contraintes 125 à $1000 \mathrm{kPa}$ semble être légèrement plus grand pour les températures de 20 à $30^{\circ}$ que pour les températures extrêmes $2^{\circ}$ et $40^{\circ}$,

\section{CONCLUSIONS}

Les résultats principaux des essais exécutés à l'œdomètre sont les suivants:

- la déformabilité du sol non saturé, caractérisée par


compression $\mathrm{C}_{\mathrm{c}}$ ne varie pratiquement pas avec la tem. pérature. Dans le cas du sable fin, la température a un très léger effet sur la compacité initiale des éprouvettes (l'influence de $\mathrm{T}$ n'est pas encore étudiée pour le limon). Lorsque le sable est non saturé, sa déformabilité est beaucoup plus grande que s'il est sec ou saturé. Dans ce cas, la déformabilité ne dépend pas de la teneur en eau entre $2,5 \%$ et $20 \%$.

La déformabilité du limon, plus faible que celle du sable, augmente lorsque la teneur en eau croît entre 10 et $20 \%$. Pour les fortes teneurs en eau, le sol se sature en cours d'essai par diminution de la porosité;

- la succion varie avec la porosité pour une même température et une teneur en eau constante. Elle augmente lorsque la porosité diminue si le degré de sa. turation $S_{r}<60$ à $70 \%$. Si $S_{r}>60$ à $70 \%$, la succion diminue avec la porosité pour s'annuler lorsque $\mathrm{S}_{\mathrm{r}}=$ $100 \%$. Les lois proposées par SEKER (1983) traduisent cette tendance. La valeur numérique des coefficients de ces lois reste à vérifier;

- l'évolution des déformations au cours du temps est bien traduite par la loi en logarithme du temps (fluage amorti). Les deux paramètres de cette loi ne varient que dans les domaines relativement étroits.

Le tassement instantané (20 premières minutes) $\varepsilon_{20}$ est compris dans l'intervalle 0,009 à 0,035 pour le sable et
0,003 à 0,025 pour le limon, quelles que soient la teneur en eau, la contrainte de compression ou la température. Le coefficient de fluage a varie entre 0,0005 et 0,0030 pour le sable, entre 0,001 et 0,009 pour le limon. La différence de comportement entre le sable et le limon est donc faible.

Du point de vue pratique, le calcul du tassement d'une fondation peut se faire à partir d'une courbe cedométrique déterminée pour le sol non saturé, sans se préoccuper des succions, c'est-à-dire en introduisant les contraintes totales. L'erreur à ne pas commettre est de saturer l'éprouvette dans l'œedomètre, comme on le fait habituellement. Cela pourrait être cause d'erreurs importantes.

L'évolution des tassements dans le temps est très sensiblement différente pour un sol non saturé et pour un sol saturé. Le calcul du degré de consolidation U après un jour ( $\mathrm{i}=1440$ minutes) dans les quatre cas du tableau VII, donne les résultats suivants:

$$
\left(\mathrm{U}=\frac{\Delta \mathrm{h}_{\mathrm{i}}}{\Delta \mathrm{h}_{\infty}}\right)
$$

Note: dans le cas saturé, les valeurs admises dans le calcul sont:

Sable: $\mathrm{k}=3,6 \cdot 10^{-5} \mathrm{~m} \cdot \mathrm{s}^{-1}$ et $\mathrm{E}_{\text {oed }}=20000 \mathrm{kN} \cdot \mathrm{m}^{-2}$ Limon : $\mathrm{k}=1,0.10^{-8} \mathrm{~m} \cdot \mathrm{s}^{-1}$ et $\mathrm{E}_{\text {ced }}=10000 \mathrm{kN} \cdot \mathrm{m}^{-2}$ L'épaisseur $\mathrm{H}=10 \mathrm{~m}$ pour les deux sols

\section{Tableau IX}

Table IX

\begin{tabular}{|l|ll|c|}
\hline \multicolumn{1}{|c|}{ Sol } & \multicolumn{2}{|c|}{ non saturé } & saturé \\
\hline Sable & Cas 1 & $U=76 \%$ & \\
& Cas 3 & $U=56 \%$ & $U=99 \%$ \\
& Cas 4 & $U=85 \%$ & \\
\hline Limon & Cas 2 & $U=39 \%$ & $U=10 \%$ \\
\hline
\end{tabular}

Le sable non saturé se déforme donc plus lentement que le même sol saturé. Dans le cas du limon, c'est linverse.

Finalement, les essais ont montré que la succion varie fortement lorsque la porosité change à teneur en eau massique constante. Elle est aussi très sensible à la température. Si cette observation n'a pas grande importance dans le cas d'un calcul de tassements, elle en aura par contre lors de calculs de stabilité de soutènements ou de pentes.

Dans la plupart des traités sur les transferts de fluides biphasiques, en milieu non saturé (voir par exemple BAER, 1979, chapitre 6.2) les équations du mouvement sont écrites en admettant deux choses:

- la matrice solide du milieu poreux est rigide, la po. rosité ne varie pas. Il n'y a ni consolidation, ni subsidence;

- la pression déterminante des fluides en mouvement est négative; elle est égale à la succion. 
Ce sont les hypothèses habituelles des hydrogéologues et des spécialistes étudiant les transferts de polluants. L'ingénieur au contraire est particulièrement intéressé par les déformations, les variations de porosité qu'accompagnent les tassements. La mécanique des sols doit donc utiliser d'autres équations du mouvement des fluides puisque nos essais ont montré la dépendance entre succion et porosité. La notion de contrainte effective est-elle valable encore en milieu non saturé, la contrainte neutre étant égale à la succion? C'est ce que devraient confirmer d'autres recherches.

\section{REMERCIEMENTS}

Nos remerciements sont adressés au Fonds National Suisse de la Recherche Scientifique (FNRS) et à l'Ecole Polytechnique Fédérale de Lausanne (EPFL) qui ont investi des moyens financiers importants pour ces recherches. Ils vont aussi à Daniel BOVET, ingénieur physicien, responsable de ce groupe de recherche au Laboratoire de mécanique des sols de l'EPFL ainsi qu'à
Mohamed EL TANI, ingénieur physicien, qui a exécuté lui-même la plus grande partie des essais et a contribué à l'exploitation de leurs résultats.

\section{BIBLIOGRAPHIE}

TERZAGHI, K. (1925), Erdbaumechanik auf Bodenphysikalischer Grundlage. Franz Deuticke. Leipzig, Wien.

LAMBE, T.W., WHITMAN, R.V. (1969), Soil Mechanics, John Wiley and Sons, inc., New York.

BEAR, J. (1972), Dynamics of fluids in porous media, American Elsevier, New York.

BEAR, J. (1979), Hydraulics of Groundwater, Mc Graw. Hill, New York.

BEAR, J. (1987), Modeling Groundwater Flow and Pol. lution, D. Reidel, Dordrecht.

SEKER, E. (1983), Etude de la déformation d'un massif du sol non saturé. Thèse $n^{\circ} 492$, présentée à l'Ecole Polytechnique Fédérale de Lausanne. 